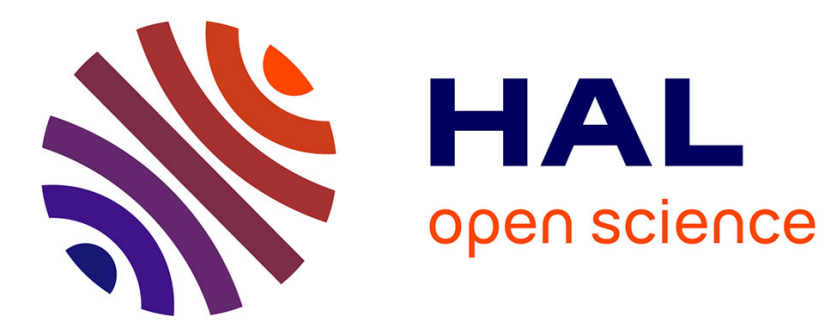

\title{
Hydrodynamic Regimes Induced by Nanosecond Pulsed Discharges in Air: Mechanism of Vorticity Generation
}

Ciprian Dumitrache, Arnaud Gallant, Nicolas Minesi, Sergey Stepanyan, Gabi Daniel Stancu, Christophe O Laux

\section{- To cite this version:}

Ciprian Dumitrache, Arnaud Gallant, Nicolas Minesi, Sergey Stepanyan, Gabi Daniel Stancu, et al.. Hydrodynamic Regimes Induced by Nanosecond Pulsed Discharges in Air: Mechanism of Vorticity Generation. Journal of Physics D: Applied Physics, 2019, 10.1088/1361-6463/ab28f9 . hal-02164353

\section{HAL Id: hal-02164353 https://hal.science/hal-02164353}

Submitted on 26 Jun 2019

HAL is a multi-disciplinary open access archive for the deposit and dissemination of scientific research documents, whether they are published or not. The documents may come from teaching and research institutions in France or abroad, or from public or private research centers.
L'archive ouverte pluridisciplinaire HAL, est destinée au dépôt et à la diffusion de documents scientifiques de niveau recherche, publiés ou non, émanant des établissements d'enseignement et de recherche français ou étrangers, des laboratoires publics ou privés. 


\section{Hydrodynamic Regimes Induced by Nanosecond Pulsed Discharges in Air: Mechanism of Vorticity Generation}

Ciprian Dumitrache, ${ }^{1)}$ Arnaud Gallant, ${ }^{1)}$ Nicolas Minesi,${ }^{1)}$ Sergey Stepanyan, ${ }^{1)}$ Gabi D. Stancu, ${ }^{1)}$ and Christophe O. Laux ${ }^{1)}$

${ }^{1}$ Laboratoire EM2C, CNRS, CentraleSupélec, Université Paris-Saclay, Eiffel Building, 3 rue Joliot-Curie, 91190 Gif-

sur-Yvette, France

The mechanisms controlling the hydrodynamic effects induced by nanosecond pulsed discharges applied between two pin electrodes in air at atmospheric conditions are investigated experimentally and numerically. A cylindrical plasma kernel is formed between the electrodes during the discharge. After the discharge, schlieren images show that the cylindrical kernel evolves in different ways depending on the gap distance: (1) for short gap distances $\left(d_{\text {gap }}<4 \mathrm{~mm}\right)$, the cylindrical kernel collapses to form a torus-like structure. In this case the flow field presents a significant source of vorticity; (2) for larger gaps $\left(d_{\text {gap }} \geq 4 \mathrm{~mm}\right)$, the kernel retains its initial cylindrical shape and cools down primarily through heat diffusion. The objective of this work is to understand the mechanisms leading to the formation of these two hydrodynamic regimes. To this end, simulations were performed and compared with the experimental schlieren images. It is shown that the main source of vorticity is the baroclinic torque caused by the cylindrical blast wave that follows the fast energy addition during the discharge. Finally, a criterion is given to predict the occurrence of the two hydrodynamic regimes. This criterion is then validated against experimental results from the literature.

\section{INTRODUCTION}

Low-temperature non-equilibrium plasmas have generated significant interest for various applications in recent years. In particular, Nanosecond Repetitively Pulsed (NRP) discharges are used for aerodynamic flow control of boundary layers[1], plasma-assisted combustion enhancement thanks to their ability to extend the flame blow-off limit [2,3], increase the flame propagation velocity [4-6], and provide flame stabilization [7,8]. To explain the observed benefits, a significant body of research has been dedicated to understanding the influence of the radicals generated in the plasma on flame propagation [7,9,10]. However, little is currently known about the hydrodynamic regimes induced by NRP discharges and their effects on combustion and aerodynamic flow control are not yet fully understood. 
Past research by Lovascio et al. [11,12] examined the effects of the pulsing frequency on the ignition delay of combustible mixtures, and reported that there is an optimal repetition rate for which the delay is minimal. It is suggested in their work that, at high pulse repetition rate, flow disturbances that persist from previous pulses are detrimental to the flame kernel development. This explanation is supported by numerical modeling work performed by Castela et al. [13], who showed that significant entrainment of fresh gas takes place after the plasma kernel collapse. This vorticity-induced flow can last for several hundreds of microseconds after the end of the discharge. The hydrodynamic effects for a single discharge pulse are also described in the work of Thiele et al. [14]. They noted the development of a recirculation zone behind the blast wave, several microseconds after the pulse, as well as the transition from an initially cylindrical shock wave front to a spherical one. Nonetheless, no explanation was provided as to the actual mechanism responsible for vorticity generation in the post-discharge.

In this paper, we investigate the hydrodynamics of NRP discharges in air at atmospheric conditions for various inter-electrode gaps. Two regimes are identified: 1) for small gaps $\left(d_{g a p}<4 \mathrm{~mm}\right)$, the hot gas kernel left after plasma recombination collapses into a symmetric torus; 2 ) for large gaps $\left(d_{\text {gap }} \geq\right.$ $4 \mathrm{~mm}$ ), a purely diffusive kernel is observed and the kernel's initial cylindrical shape is maintained throughout the cooling phase. These experimental observations are further confirmed and explained by analyzing the flow-field around the discharge using computational fluid dynamics simulations. Additionally, the main mechanism through which vorticity is generated is identified to be a baroclinic torque resulting from a misalignment between the density and pressure gradients at the kernel interface.

This manuscript discusses the mechanism of vorticity generation by nanosecond discharges. To this end, high-speed schlieren imaging is employed to follow the post-discharge kernel development in the two different hydrodynamic regimes described earlier and CFD modeling is used to simulate the observed phenomena. The main mechanisms of vorticity generation are identified, and a physical interpretation of the observed flow-field is given. Finally, a dimensionless parameter that predicts the transition between the two regimes is proposed.

\section{METHODS}

\section{A. Experimental Setup}

NRP discharges are produced between two pin electrodes made from 2-mm diameter tungsten rods

that are mechanically-sharpened to achieve a tip electrode radius $\sim 150-300 \mu \mathrm{m}$. A high-voltage pulse 
generator (FID FPG20-30MKS50) is used to provide the electric energy required for breakdown. The generator can deliver pulses of up to $30 \mathrm{kV}$, with a pulse length tunable between 10-50 ns at a maximum repetition rate of $30 \mathrm{kHz}$. The discharge takes place inside a custom designed reactor able to operate over a wide pressure range ( $\mathrm{p}=0.001$ - 10 bar). The electric energy coupled into the plasma is determined for various inter-electrode gaps. The voltage across the electrodes is measured with a $100-\mathrm{MHz}$ bandwidth high-voltage probe (Lecroy PPE20kV) while the discharge current is measured with a Pearson coil current monitor (Model 6585) with a 1.5-ns rise time and $1 \mathrm{~A} / \mathrm{V}$ current-to-voltage conversion factor when terminated with a $1 \mathrm{M} \Omega$ impedance. The current and voltage signals are recorded using a $1 \mathrm{GHz}$ LeCroy Wavepro 7000 oscilloscope. The electric energy is determined by integrating the product of the voltage and current waveforms over the pulse duration. The actual energy deposited into the plasma is determined as the difference between the incoming and reflected pulse energy (reflection arises due to impedance mismatch at the inter-electrode gap). This method assumes that the losses through heating of the electrodes and radiation (through the electrode antenna) are negligible [15]. These assumptions were checked by repeating the experiment at high pressures, where no breakdown was present, and the reflected energy matched the incoming energy within 5\%. Figure 1 shows a diagram of the electric measurement setup and a sample plot of the measured current, voltage, and energy waveforms. Note that the oscillogram presented below does not represent the actual voltage and current traces used in the current experimental study and it only serves as an example to show how the energy deposited is extracted from the voltage and current data. The traces from Fig. 1-right correspond to electrical measurements performed in the middle of a $75 \Omega$ cable (a voltage pulse of $\sim 4 \mathrm{kV}$ corresponding to a $\sim 50$ A current as seen in Figure 1-left). However, in the experiments presented in the next sections, the pulser was configured in an impedance matching configuration. The actual voltage supplied was set to $30 \mathrm{kV}$ giving a current of $100 \mathrm{~A}$ (based on the internal impedance of the generator of $300 \Omega)$. 



Figure 1: (Left) Schematic of the experimental setup used for electrical measurements in NRP discharges. (Right) Sample oscillogram showing the current and voltage measured by the probes as well as the calculated electrical energy. The probes were placed in the middle of a $75 \Omega$ cable.

The hydrodynamic development of the plasma kernel over time is observed experimentally using a high-speed laser-illuminated schlieren setup. The optical layout is shown in Figure 2. Briefly, a 2-mW He:Ne laser (Thorlabs HNL020LB) operating at $632.8 \mathrm{~nm}$ is employed as a light source. The laser beam is expanded using a negative lens $(\mathrm{f}=-35 \mathrm{~mm})$ to overfill the aperture of a 2-inch collimation lens $(\mathrm{f}=1000 \mathrm{~mm})$. This is done to achieve an evenly illuminated background for the schlieren images. The collimated light is then passed through the discharge reactor using a pair of 2-inch silver-coated mirrors. After leaving the reactor, the beam is focused onto a partially blocking knife-edge using a plano-convex lens $(\mathrm{f}=500 \mathrm{~mm})$. In order to reduce the diffraction effects induced by the coherent light source, a neutral density graded knife edge is used at the cutoff plane instead of the typical razor blade employed in schlieren setups illuminated by white light sources [16,17]. Finally, the light is collected using a f/6.0 lens onto a CMOS high-speed camera (Photron Fastcam SA-X2). Images are collected at a repetition rate of $100 \mathrm{kHz}$ and over 315 × 256 pixels $(20 \mu \mathrm{m}$ x $20 \mu \mathrm{m}$ pixel size $)$. 


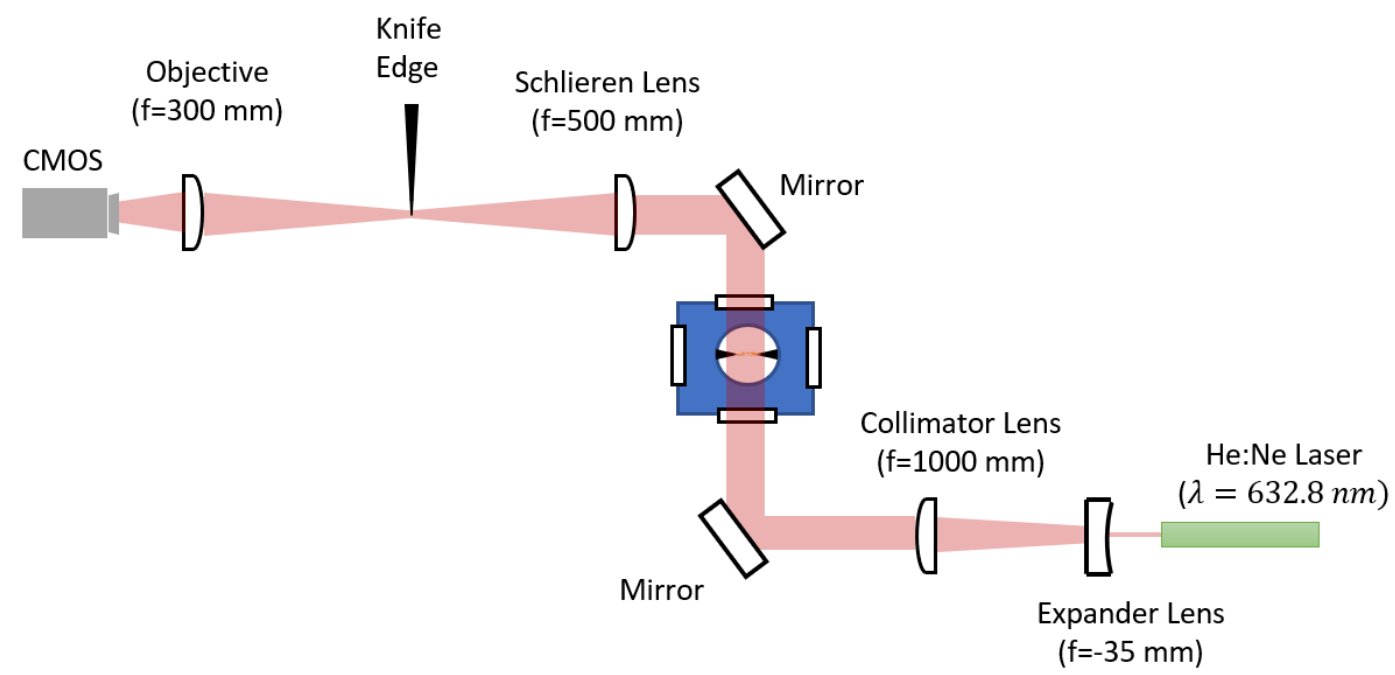

Figure 2: Optical layout for high-speed schlieren imaging of NRP discharges in air at atmospheric conditions ( $\mathrm{p}=1 \mathrm{bar}$, $\mathrm{T}=300 \mathrm{~K})$.

\section{B. Numerical Model}

The fluid dynamics generated by the discharge were simulated using a custom-built Navier-Stokes solver [18]. For the purpose of this study, the plasma chemical kinetics are not considered in the model. Instead, the model focuses primarily on the hydrodynamics induced by the plasma produced in the electrode gap after the end of the discharge (i.e. simulations begins after the end of the discharge). This simplification of the model is justified because the kernel collapse and vorticity generation are observed several microseconds after the pulse, i.e. much later than the end of the discharge pulse

The governing equations written in cylindrical coordinates with axial symmetry (and zero azimuthal velocity) are presented below in conservative form:

$$
U_{t}+F(U)_{r}^{a}+G(U)^{a}{ }_{z}=F(U)_{r}^{d}+G(U)^{d}{ }_{z}+S(U)
$$

where:

$$
U=\left[\begin{array}{c}
\rho \\
\rho u \\
\rho v \\
E
\end{array}\right] ; \quad F(U)^{a}=\left[\begin{array}{c}
\rho u \\
\rho u^{2}+p \\
\rho u v \\
u(E+p)
\end{array}\right] ; \quad G(U)^{a}=\left[\begin{array}{c}
\rho v \\
\rho u v \\
\rho v^{2}+p \\
v(E+p)
\end{array}\right]
$$




$$
\begin{gathered}
F(U)^{d}=\left[\begin{array}{c}
0 \\
\tau_{r r} \\
\tau_{r z} \\
u \tau_{r r}+v \tau_{r z}-q_{r}
\end{array}\right] ; \quad G(U)^{d}=\left[\begin{array}{c}
0 \\
\tau_{r z} \\
\tau_{z z} \\
u \tau_{r z}+v \tau_{z z}-q_{z}
\end{array}\right] ; \\
S(U)=-\frac{1}{r}\left[\begin{array}{c}
\rho u \\
\rho u^{2}-\tau_{r r} \\
\rho u v-\tau_{r z} \\
u(E+p)+u \tau_{r r}-v \tau_{r z}+q_{r}
\end{array}\right] ;
\end{gathered}
$$

In Eq. (2), $U$ is the vector of conservative variables, $F(U)^{a}$ and $G(U)^{a}$ describe the advection fluxes (in the $\mathrm{r}$ and $\mathrm{z}$ direction, respectively), $F(U)^{d}, G(U)^{d}$ represent the diffusion fluxes, and $S(U)$ is the source term that is the result of the coordinate system transformation from cartesian to cylindrical. $\rho$ is the mass density, $p$ the pressure, $u$ and $v$ the speed components in the $r$ (radial) and $z$ (axial) directions, respectively, $E$ the total energy of the system (i.e. the sum of internal and kinetic energies), $\tau_{\mathrm{ij}}$ the shear stress components, and $q_{i}$ the conductive flux components.

The system of conservation equations is complemented with two additional relations that describe the thermodynamic state of the system:

$$
\begin{gathered}
p=\rho R_{g} T \\
E=\rho\left(h-\frac{p}{\rho}+\frac{u^{2}+v^{2}}{2}\right)
\end{gathered}
$$

where $R_{g}$ is the specific gas constant and $h$ the specific enthalpy. Because the calorically perfect gas assumption $\left(c_{p}, c_{v}=\right.$ const. $)$ does not apply in the case of the high temperature gas kernels generated by the discharge, the numerical model only assumes a thermally perfect gas. An implicit nonlinear equation is then solved for temperature in each computational cell and at each time step. The following relation for temperature is derived from Eqs. (3) and (4):

$$
T=\frac{-E+\rho\left(\frac{u^{2}+v^{2}}{2}\right)+\rho h(T)}{\rho R_{g}}
$$

In Eq. (5) the specific enthalpy, $h(T)$, is computed using the Shomate interpolation polynomials according to the NIST database [19]. The shear stress components are calculated using Sutherland's law, assuming a Newtonian fluid with temperature-dependent viscosity, and the conductive fluxes are calculated with temperature-dependent thermal conductivities [20]. 
From a numerical point of view, the conservation equations are solved using a dimensional splitting method. The advection terms are solved using Roe's flux differencing scheme with slope limiters [21,22] and the diffusion terms are solved using a second order centered difference scheme [23]. Finally, the resulting ODEs are integrated with an explicit fourth order Runge-Kutta method.

\section{Numerical Setup}

The generation of physical initial conditions is critical in the successful modeling of the NRP hydrodynamics. Ideally, one would measure the pressure and temperature (or density) of the kernel at the end of the pulse. However, this is challenging in practice. Instead, the pulse energy deposited into the plasma determined from the electrical measurements is used to generate initial pressure and temperatures profiles. A phenomenon of ultrafast heating by nanosecond discharges in air was first observed by Pai et al. [24,25]. Past research focusing on the kinetics of the discharge has identified the main channels that lead to gas heating. Popov [26], has suggested for air discharges at reduced electric fields around $100 \mathrm{Td}$ that a two-step dissociative quenching mechanism of excited molecular nitrogen species (created in the first step by electron impact reactions) leading to oxygen dissociation is the dominant mechanism for gas heating in nanosecond discharges. His kinetic model suggests that approximatively $20 \%$ of the total electric energy goes into ultrafast gas heating through this channel. This percentage is further confirmed experimentally by Rusterholtz et al. [27] through optical emission spectroscopy (OES) measurements inside the plasma, the rest of energy being dissipated into dissociation, formation of metastable and vibrational excited states [28,29]. For higher reduced electric field, the energy goes mainly into ionization and dissociation and much less into electronic excitation of molecules. Recent results by Lo et al. [30] and by Minesi et al. [31] showed that at E/N above $400 \mathrm{Td}$ the main plasma emission comes from atomic ions. Thus, the mechanism of ultrafast heating responsible for the formation of blast wave is different at high $\mathrm{E} / \mathrm{N}$.

Similar to Castela et al. [13], and in agreement with the measurements performed by Rusterholtz et al. [27], we assumed $20 \%$ of the measured energy going into ultrafast heating for all inter-electrode gap cases simulated here. This assumption was supported by the best match of the dynamics observed experimentally (moment of shockwave detachment and propagation, kernel size). The energies in Figure 3-left were converted into heat and we determined the initial (at the end of discharge) temperature using:

$$
\Delta E=m c_{v}(T) \Delta T
$$


where $\Delta E$ corresponds to $20 \%$ of the total energy deposited into the gas. It is important to note that, while most of the energy deposited ultimately goes into heating, the ultrafast heating mechanism is the one responsible for the generation of shock waves and the ensuing hydrodynamics. Secondary heating mechanisms, such as heating generated through V-T relaxation reactions or quenching of metastable atomic states, take place on a much longer temporal scale (long after shock wave detachment and vorticity generation in the fluid) and they can be ignored for the purpose of this study. Once the temperature is determined, the pressure is computed using the ideal gas equation of state while keeping $\rho$ constant. This assumption remains correct as long as the discharge pulse length is much shorter than the hydrodynamic time scale, which is given by the kernel diameter divided by the speed of sound (i.e. $\sim \mu \mathrm{s})$.

The measured pulse energy added into the plasma, determined by the method outlined in Section IIA, is shown in Figure 3-left. For all inter-electrode gaps, a 10-ns pulse (full-width half-maximum) was used and the voltage was set to $\mathrm{U}=30 \mathrm{kV}$ in a matched load configuration giving a current $\mathrm{I}=100 \mathrm{~A}$ (based on the internal impedance of the generator of $300 \Omega$ ). The initial temperature and pressure determined using the method outlined above for the CFD simulations are presented in Figure 3-right for various inter-electrode gaps.


Figure 3: (Left): Experimentally measured discharge energy as a function of the electrode gap. (Right): Corresponding initial gas temperature (dots) and pressure (squares) at the center of the discharge calculated from the experimental data for various electrode gaps. The calculated temperatures and pressures were obtained by using the average energy at each inter-electrode gap from the left plot.

The discharge kernel is assumed to be initially a cylinder, of height equal to the inter-electrode gap distance. Additionally, spatially resolved optical emission and schlieren measurements indicate that the discharge radius is $\sim 100 \mu \mathrm{m}$ at the end of the discharge pulse [32,33]. Changing the inter-electrode gap 
was shown to have very little impact on the discharge radius. Thus, a fixed kernel radius of $100 \mu \mathrm{m}$ was used for all the inter-electrode gaps studied here.

The shape of the initial kernel on the computational grid is shown in Figure 4. To obtain the initial profiles, a gaussian blending filter was used to generate a smooth variation of the temperature and pressure profiles from their maximum value at the center of the kernel to ambient conditions ( $p=1 \mathrm{bar}$, $T=300 \mathrm{~K}$ ) in the far-field (see Figs 3 and 4). Since the energy is deposited over a very short time period (discharge pulse is much shorter than the acoustic time-scale), the gas density remains unchanged at the end of the discharge. Therefore, the initial temperature and pressure profiles are assumed to have the same initial shape. The presence of the electrodes is ignored in the current model and the cylindrical kernel is assumed to propagate in atmospheric air. This model simplification is supported by past modeling work by Kono et al.[34] and by Castela et al.[13], which showed that the absence of the electrodes does not affect the hydrodynamic effects observed during the kernel cooling phase.

The governing equations are solved on a uniform structured Cartesian grid using a control volume discretization approach. A piece-wise distribution of initial data over the computational cells is employed where the value at the center of a cell represents the average of each variable over the cell. It is important to note that the domain size is chosen such that the cell size of $15 \mu \mathrm{m}$ is preserved irrespective of the kernel aspect ratio. The optimal cell size was determined through a grid sensitivity study in which various cell sizes (between 5-100 $\mu \mathrm{m}$ ) were tested. In the current configuration, the cell size is constant throughout the entire computational domain. Nonetheless, since the kernel has both radial and axial symmetry, only a quarter of the domain is modeled. Further simplification is obtained with the use of non-reflective boundary conditions at the edge of the domain, which allows the evacuation of the blast wave with minimum disturbance into the computational domain $[35,36]$. 


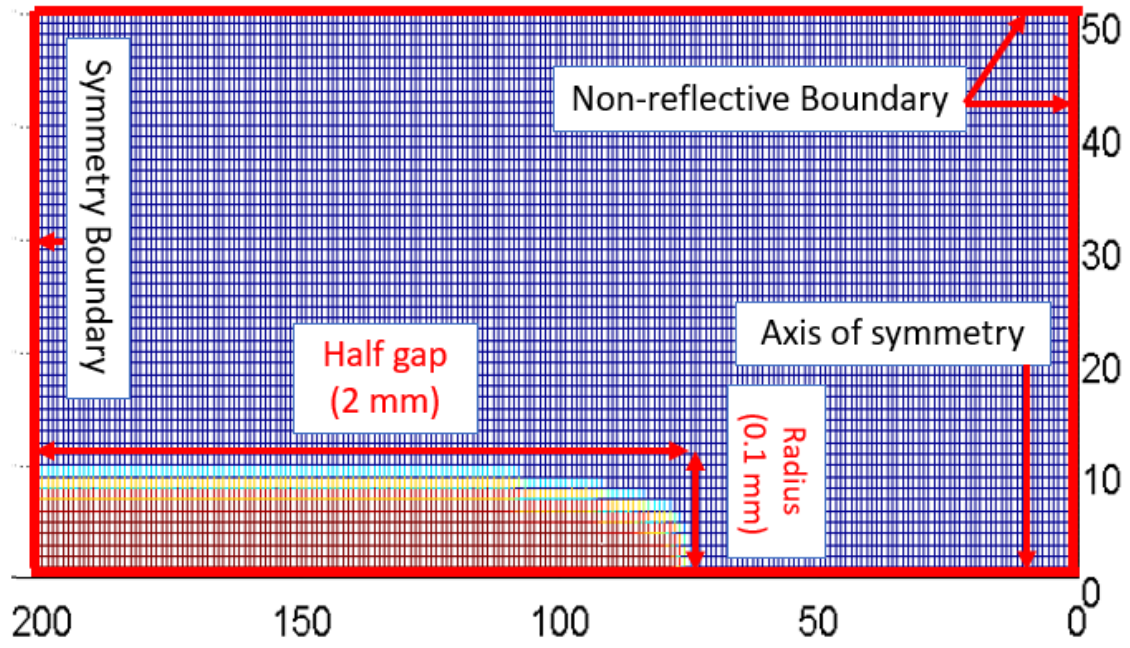

Figure 4: Initial temperature and pressure profiles on the computational grid. The case shown here corresponds to a 4-mm inter-electrode gap and a computational grid size of 50 x 200 cells.

\section{RESULTS \& DISCUSSIONS}

\section{A. High-Speed Schlieren of NRP Discharges}

The dynamics induced by the discharge are first examined using the high-speed schlieren setup described in Section II-A. Figure 5 shows two cases: a) small inter-electrode gap of $1 \mathrm{~mm}$ (Figure 5top); b) large inter-electrode gap of $4 \mathrm{~mm}$ (Figure 5-bottom). As the inter-electrode gap is changed, the hydrodynamic evolution of the hot gas kernel produced by the discharge varies significantly. For the small gaps $\left(d_{\text {gap }}=0.5-3 \mathrm{~mm}\right)$, the initially cylindrical kernel transforms into a symmetric torus. As time evolves, the hot gas is pushed into the side lobes (as seen in the schlieren images of Figure 5) by entrainment of the cold surrounding gas. Depending on the inter-electrode gap size and energy deposited, the torus can take between several tens of microseconds to several hundreds of microseconds to dissipate. In the case shown here, the inter-electrode gap was $1 \mathrm{~mm}$ and the energy deposited was $\sim 1$ $\mathrm{mJ}$. Under these conditions, the kernel fully dissipates in $\sim 150 \mu$ s (i.e. no significant disturbances are observed in the schlieren images at later times). For large gaps $\left(d_{\text {gap }} \geq 4 \mathrm{~mm}\right)$, the cylindrical kernel never collapses. Instead, one observes a purely diffusive kernel that slowly dissipates into the surroundings as time evolves. Another important difference between the two kernels is given by the strength of the blast wave which is much stronger in the $1 \mathrm{~mm}$ inter-electrode gap case as observed by the contrast in the schlieren images at $t=2 \mu \mathrm{s}$. This suggests that the temperature (and consequently the pressure) increase produced by the discharge is smaller for the $4 \mathrm{~mm}$ gap case, causing a much weaker blast wave. 



$(2 \mu s)$
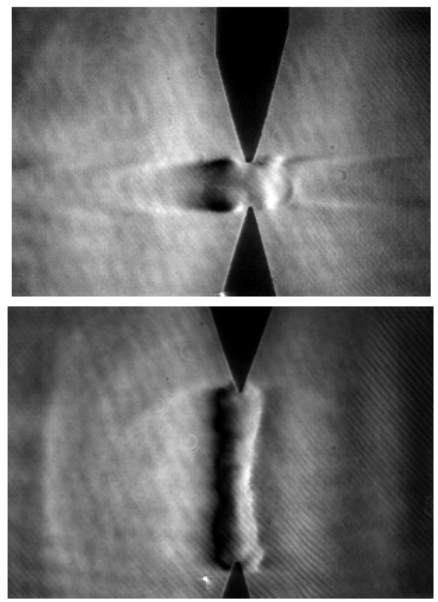

$(12 \mu s)$
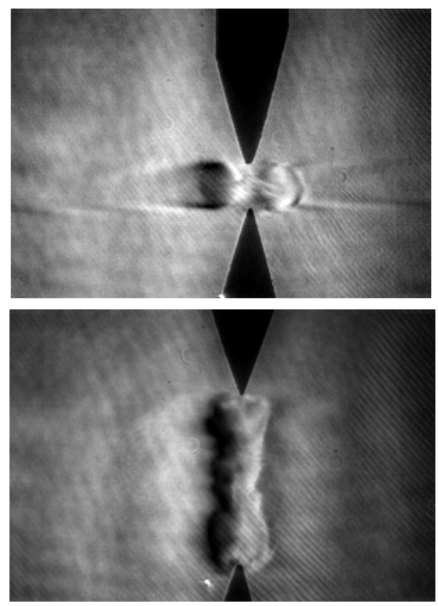

$(22 \mu s)$
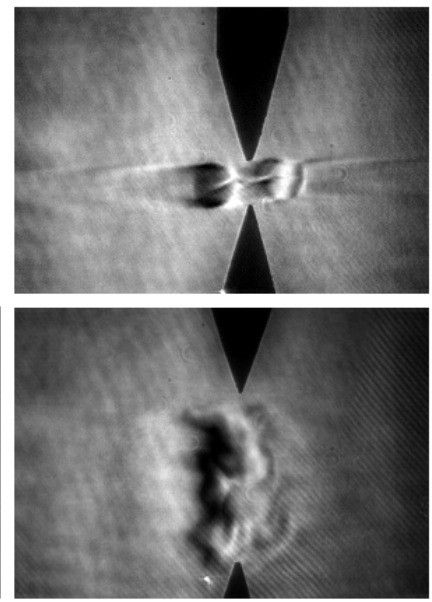

$(32 \mu s)$

Figure 5: High-speed schlieren images showing the evolution of the plasma kernel for $\mathrm{d}=1 \mathrm{~mm}$ (top) and d=4 mm gap (bottom). The images show that, as the inter-electrode gap is changed, the hydrodynamic regimes are different, with a torus created at small gaps and a diffusive kernel at large gaps. Each image frame has a field of view of $6 \mathrm{~mm} \times 9 \mathrm{~mm}$.

\section{B. Numerical Modeling}

The experimental findings presented above motivated the numerical study of the two different hydrodynamic regimes induced by the NRP discharge. Computational modeling results for the same two gaps are shown in Figure 6. The code simulates the kernels at the end of the discharge when the pressure and temperature at the core of the kernel were estimated to be: $\mathrm{T}_{1 \mathrm{~mm}}=6000 \mathrm{~K}, \mathrm{P}_{1 \mathrm{~mm}}=21.1$ bar and $\mathrm{T}_{4 \mathrm{~mm}}=4700 \mathrm{~K}, \mathrm{P}_{4 \mathrm{~mm}}=16.5$ bar, respectively. Synthetic schlieren images obtained by plotting the contours of the numerically computed density gradient are compared with the experiments. For the 1- mm gap, the kernel collapse determined numerically closely follows the experimental observation. For the 4-mm gap, the code simulates a perfectly symmetrical kernel that slowly diffuses. This is slightly different from the one observed experimentally. In reality, the discharge kernel is not perfectly axisymmetric for large gaps. Moreover, the existence of Rayleigh-Taylor instabilities into the gas at later times break the kernel symmetry [37]. Nonetheless, the main dynamics are captured in both scenarios and the code predicts well the transition from a torus to a diffusive kernel as the inter-electrode gap is increased. The blast wave is also shown to be much weaker for the 4-mm gap case, being barely visible at $2 \mu \mathrm{s}$ after the discharge. 

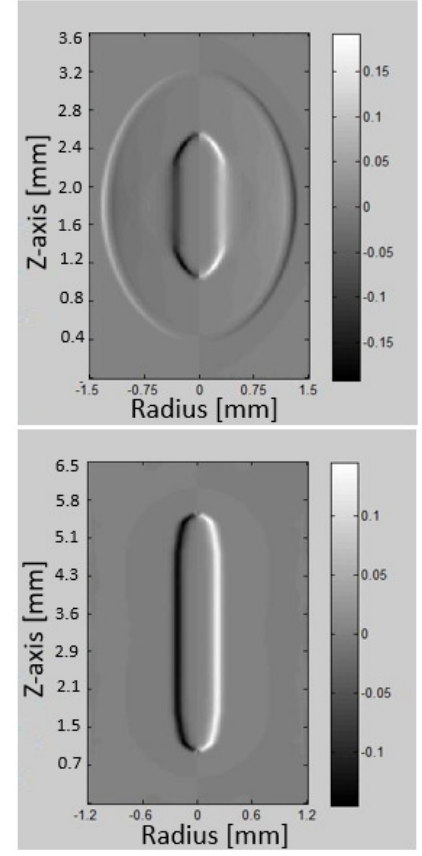

$(2 \mu s)$



$(12 \mu s)$


$(22 \mu s)$
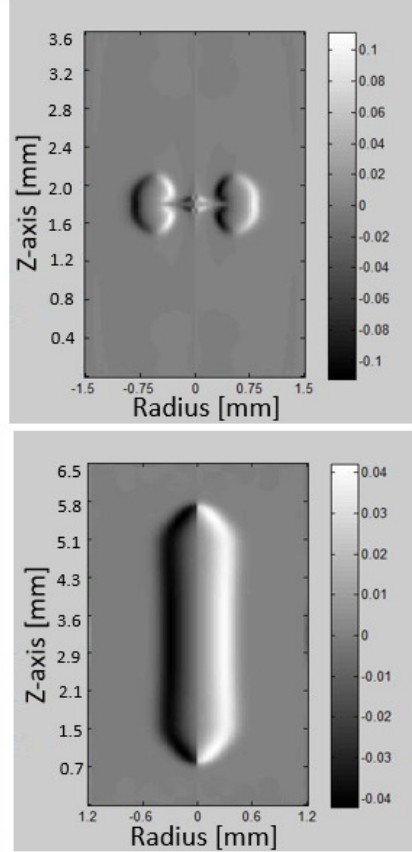

(32 $\mu s)$

Figure 6: Synthetic schlieren images showing the hot gas kernel evolution for two inter-electrode gaps: $1 \mathrm{~mm}$ (top) and 4 mm (bottom).

\section{Physics of Vorticity Generation}

To understand the physical mechanism driving the transition from one hydrodynamic regime to another the velocity field was studied during the plasma kernel cool-down phase. The velocity field of the kernel formed in the $1 \mathrm{~mm}$ case shows the presence of vorticity as early as $1 \mu \mathrm{s}$ after the end of the discharge (see Figure 7-left). The presence of eddies at this instant is surprising as one might expect that vorticity is induced in the flow only during the kernel collapse phase which takes place several microseconds later.

The source of flow rotation is revealed by inspecting the vorticity equation, obtained by taking the curl of the momentum equation [38]:

$$
\frac{D \vec{\omega}}{D t}=(\vec{\omega} \cdot \vec{\nabla}) \vec{u}-\vec{\omega}(\vec{\nabla} \cdot \vec{u})+\frac{1}{\rho^{2}}[\vec{\nabla} \rho \times \vec{\nabla} p]+v \vec{\nabla}^{2} \vec{\omega}
$$

The left-hand side is the material derivative of the vorticity. The first term on the right-hand side describes the enhancement of vorticity by stretching and is the mechanism through which turbulent eddies transfer energy to smaller scales. This term is zero in 2-D flows. However, the kernel collapse and the formation of the toroid is observed even if the model is run in 2-D (corresponding to setting $S(U)=0$ in Eq. 1), so this term is not the primary mechanism responsible for vorticity generation. The 
divergence of velocity in the second term corresponds physically to a volumetric expansion. Thus the vorticity decreases as the fluid expands (due to the negative sign). However, at the early stages following the discharge, the kernel is expanding and therefore this term does not contribute to vorticity generation. The fourth term in Eq. 7 describes the effects of viscous diffusion on vorticity generation. However, the contribution of diffusive fluxes at early times is minimal, the flow being primarily dominated by the pressure gradients (compressible inviscid). This leaves the third term, which describes vorticity generated by a baroclinic instability in the flow. Physically, this term is created by a misalignment between the density and pressure gradients that induces baroclinic torque. Indeed, if the pressure and density gradients are plotted in the region where flow vorticity is observed it becomes apparent that the pressure and density gradients are not aligned (see Figure 7-right).

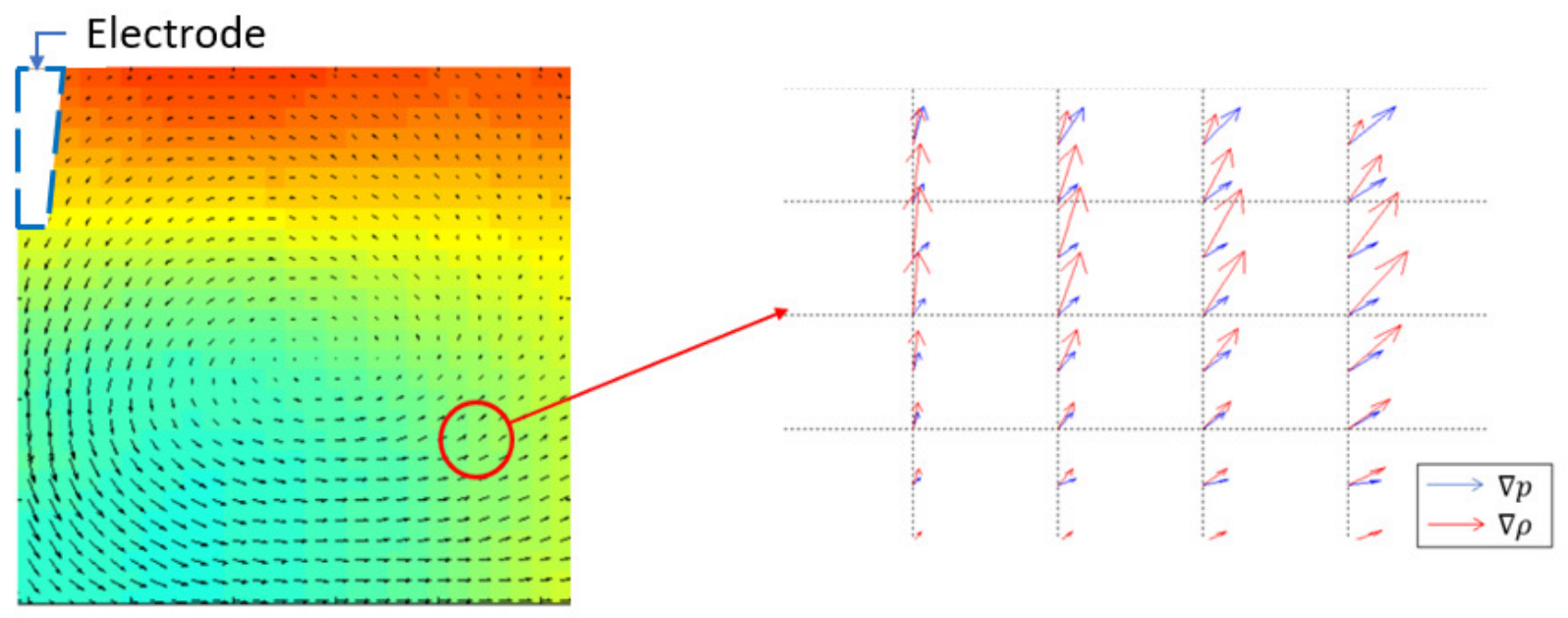

Figure 7: (left) Velocity field at $1 \mu$ s after the discharge over-imposed on density contours in the computational domain quadrant. (right) Density and pressure gradients inside the flow are not aligned, giving rise to vorticity generation by the baroclinic torque. The profiles correspond to the simulated $\mathrm{d}=1 \mathrm{~mm}$ inter-electrode gap case.

To quantify the importance of the various terms in Eq. (7), their contributions to the rate of vorticity generation are presented in Figure 8. The rate of vorticity generation reaches a maximum immediately after the blast wave separates from the kernel ( $2 \mu \mathrm{s}$ for $\mathrm{d}=1 \mathrm{~mm}$ case $)$. At this early stage, the main contributor to vorticity generation is the baroclinic torque which accounts for more than $85 \%$ of the total rate of vorticity generated in the domain. The remaining vorticity is generated by the viscous mechanism $(\sim 13 \%)$ and by the collapse of the kernel ( $>$ \%) through the second term on the right side of Eq. (7). The source baroclinic torque is quickly reduced as the blast wave moves away from the kernel and no significant sources of vorticity are observed at times longer than $20 \mu \mathrm{s}$. The curves presented in Fig. 8 
were obtained by tracking the zone of maximum vorticity generation at each time delay inside the computational domain and averaging over a 20x20 cell region around the maximum.

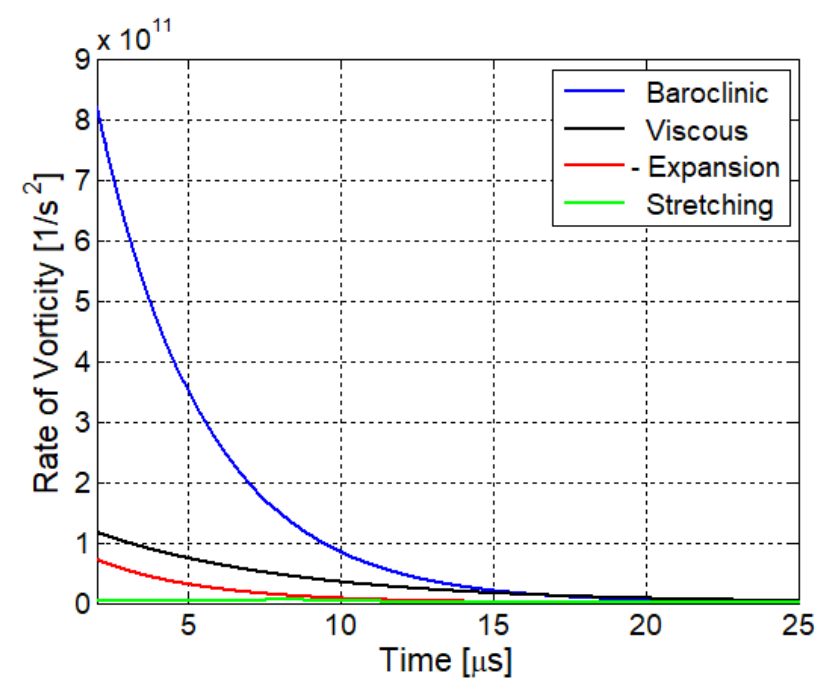

Figure 8: Contribution of each term in the vorticity equation to the rate of vorticity generation as a function of time for an inter-electrode gap corresponding to: $\mathrm{d}=1 \mathrm{~mm}$.

A similar mechanism has also been identified as being responsible for vorticity generation in laser induced sparks [39]. The initial profile of a laser spark resembles a teardrop, with the point of maximum kernel temperature having an offset towards the laser beam propagation direction. In such a case, numerical simulations showed that baroclinic torque leads to the formation of an asymmetric torus (a socalled three lobe structure) in which a gas jet is expelled through the center of the torus and propagates towards the laser source. This type of kernel has been widely reported in the laser ignition literature [4042].

The presence of baroclinic instabilities in NRP discharges was mentioned in the past $[43,44]$. However, the mechanism through which it is generated in this type of discharges was not described. A physical explanation on how baroclinic torque is generated follows from the analysis of the blast wave that accompanies the breakdown process (see schematic in Figure 9). The cylindrical shape of the kernel leads to the formation of an initially cylindrical blast wave in which the pressure gradient is not equally strong along the entire circumference. The initially cylindrical shock wave can be thought as a combination of a normal shock wave front at the sides of the kernel and two spherical shock wave fronts near the anode and the cathode. As the shock propagates, the normal shock tends to propagate faster than the spherical front. This is because a significant portion of the spherical front energy is spent on stretching the shock front (increase in perimeter) with time. Therefore, the pressure is higher along the normal front than along the spherical front. This induces a misalignment between the density and 
pressure gradients at the corners of the kernel as shown in Fig. 9, giving rise to the baroclinic torque. Given enough time, the blast wave becomes spherical and the source of the baroclinic torque vanishes.



Figure 9: Schematic showing how the evolution of the blast wave that follows breakdown leads to a baroclinic instability.

The flow-field differences between the two hydrodynamics regimes at later times are shown schematically in Figure 10. The rarefaction/expansion waves that follow the leading shock generate a velocity field directed towards the center of the domain in order to equilibrate the pressure. The lower density fluid (hotter fluid) will be accelerated more in this negative pressure gradient than the fluid at high density (colder fluid), thus generating vorticity. If the kernel aspect ratio is low, the two main sources of vorticity are located at the top and bottom edges of the kernel, as shown in Figure 10-left. However, if the kernel is elongated (which corresponds to large inter-electrode gaps), additional sources of vorticity are observed at the center of the kernel. The kernel will appear to maintain its shape if the two sources of vorticity (green and orange in Figure 10-right) are of equal strength. In reality, they are never exactly equal so the kernel tends to slowly contract or expand. If the thermal conduction time scale is much shorter than the transport generated by the mismatch between the two vorticity sources, the kernel will appear purely diffusive in nature. 


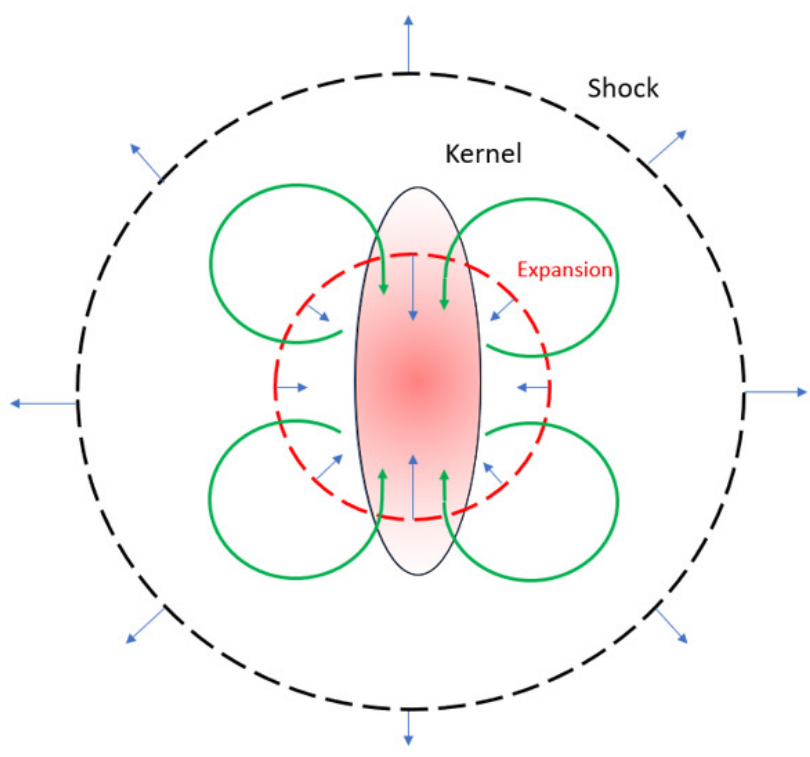

(a)



(b)

Figure 10: Schematic diagrams showing the influence of the blast wave on the generation of vorticity. For small aspect ratio kernels, (a), vorticity is generated primarily at the top and bottom edges of the kernel. For elongated kernels, (b), additional vorticity is generated along the plane of symmetry due to the elongation of the blast wave.

\section{Factors influencing the transition}

At this stage it is important to understand whether the transition is purely a geometric effect (caused only by the change in the kernel's aspect ratio) or whether it also depends on other parameters such as the energy deposited during the discharge. Presented below are experimental results performed for a fixed inter-electrode gap (d=3 mm) and two different energies: $1 \mathrm{~mJ}$ (Fig. 11-top) and $3.2 \mathrm{~mJ}$ (Fig. 11bottom). For the low energy case a slow vertical collapse is observed. However, the kernel maintains its initial cylindrical shape until it dissipates completely through Rayleigh-Taylor instabilities at several hundred of microseconds after the pulse. For the higher energy case, the kernel is noticeably bigger even at early times (see Fig. 11-bottom, panel corresponding to $t=5 \mu$ s after the pulse). This is because the kernel is hotter, which decreases the density of the gas and leads to a more efficient kernel expansion behind the blast wave. As time progresses, the kernel collapses to form a torus between the electrodes. Please note that the kernel development in this case is much slower than reported earlier for the $\mathrm{d}=1 \mathrm{~mm}$ case. Instabilities in the flow have distorted the edges of the torus at $200 \mu$ s making it much less defined than the one observed in Fig. 5-top for $\mathrm{d}=1 \mathrm{~mm}$. 

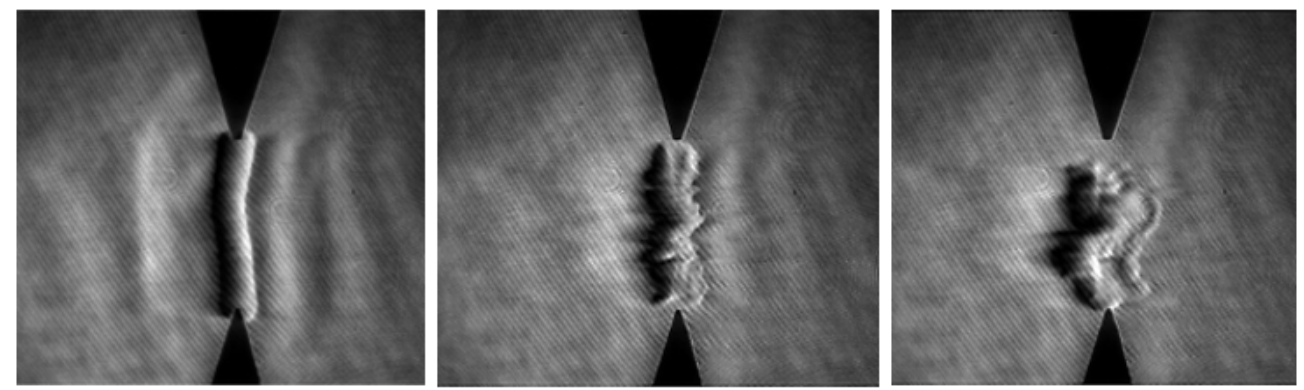



$(5 \mu \mathrm{s})$

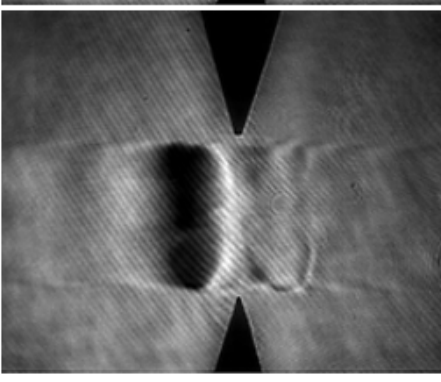

$(35 \mu \mathrm{s})$

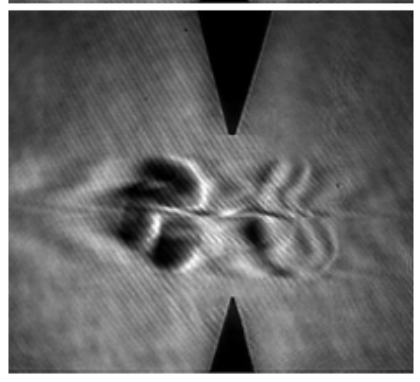

$(200 \mu \mathrm{s})$

Figure 11: Experimental schlieren images of an NRP discharge in air inside of a d=3 $\mathrm{mm}$ gap at $\mathrm{E}=1 \mathrm{~mJ}$ (top) and $\mathrm{E}=3.8 \mathrm{~mJ}$ (bottom). Each image frame has a field of view: $6 \mathrm{~mm} \times 9 \mathrm{~mm}$.

The numerical model becomes useful in this context as one can easily simulate a wider range of energies for a fixed inter-electrode gap and observe the hydrodynamic development. Shown in Figure 12 are temperature contour plots for three different energies $(\mathrm{E}=0.2 \mathrm{~mJ}, \mathrm{E}=1 \mathrm{~mJ}$ and $\mathrm{E}=5 \mathrm{~mJ})$ and a fixed gap of $3 \mathrm{~mm}$. The $5 \mathrm{~mJ}$ case is very similar to the case observed experimentally for a gap of $3 \mathrm{~mm}$ and the kernel is predicted to collapse in this case. Indeed, the same hydrodynamic regime is predicted by the CFD simulation also (see Figure 12-bottom). Interestingly, however, if the energy deposited is reduced below $1 \mathrm{~mJ}$ one can bring even a $3 \mathrm{~mm}$ gap kernel into the diffusive hydrodynamic regime (Figure 12-top). For intermediate energies (Figure 12-center) the kernel is seen to slowly collapse but it will typically diffuse before it can form a torus as confirmed by the experimental results presented above. 

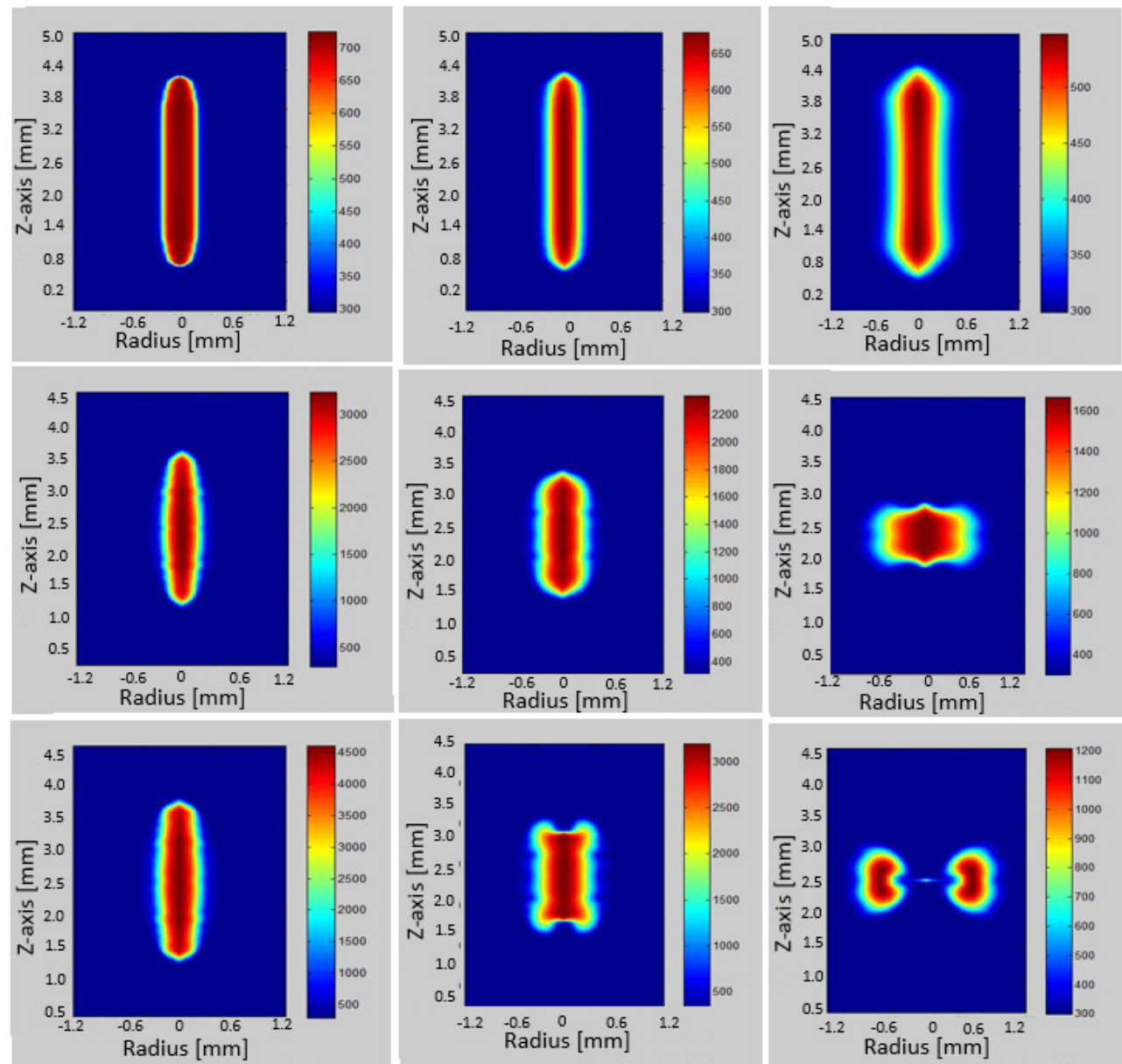

$(5 \mu s)$

$(35 \mu s)$

$(200 \mu s)$

Figure 12: Temperature contour plots (in kelvin) showing the evolution of the hot gas kernel inside a $3 \mathrm{~mm}$ inter-electrode gap at three different discharge energies: $0.2 \mathrm{~mJ}$ (top), $1 \mathrm{~mJ}$ (middle) and $5 \mathrm{~mJ}$ (bottom).

As discussed above, the hydrodynamic regime observed in nanosecond discharges depends on the energy deposited into the gas during the discharge and on the aspect ratio of the kernel. This motivates the search for a dimensionless parameter capable of predicting the transition. Such a number should include the fractional energy deposited into the kernel by ultra-fast heating, $E_{u h}$, the interelectrode gap distance, $d$, the initial radius of the kernel, and a state variable describing the initial state of the surrounding gas. The proposed number, found by dimensional analysis, is the following:

$$
\Pi_{T r}=\frac{E_{u h}}{p_{0}} \frac{1}{S d}
$$

For discharges in preheated gas, it can also be expressed in terms of the gas density and temperature using the equation of state: 


$$
\Pi_{T r}=\frac{E_{u h}}{\rho_{0} R_{g} T_{0}} \frac{1}{S d}
$$

where $R_{g}$ stands for the gas constant, $S=\pi r^{2}$ is the cross-sectional area of the cylindrical kernel and $\rho_{0}, T_{0}, p_{0}$ are the density, temperature and pressure of the surrounding gas.

In Figure 13, this dimensionless number is plotted as a function of the interelectrode gap distance for several experimental cases. Two distinct regimes can be identified in Figure 13. For small gaps ( $d<4 \mathrm{~mm}$ ), the number is relatively constant. This corresponds to the configurations where the formation of a toroidal structure in the post-discharge has been both observed experimentally and predicted by CFD modeling. For larger gaps $(d \geq 4 \mathrm{~mm})$, where a diffusive kernel is observed, the number gradually decreases with the gap distance. The linear nature of this decrease suggests that the hydrodynamics are controlled by volumetric energy addition into the gas. This hypothesis is further verified when comparing this result with Fig. 3 where it was shown that, for gaps below $4 \mathrm{~mm}$, the increase in interelectrode gap distance is compensated by an equivalent increase in energy deposited into the kernel. As a result, the dimensionless number is virtually unchanged, and the hydrodynamic regime is the same. However, further increasing the interelectrode gap distance was observed to reduce the amount of energy deposited, leading to a significant decrease of the volumetric energy. We also see that the transition from one regime to the other is gradual. Both experiments and CFD showed that there is a region between $\Pi_{t r}=60-80$ where the kernel collapse into a toroid slows down considerably. Depending on the repetition rate at which the discharge is operated, synergistic effects between separated pulses could lead to instabilities causing the kernel to shift from a toroidal to a diffusive decay.

Also included in Figure 13 are experimental results obtained from previous studies with NRP discharges. The results from Lefkowitz et al. [45] and Salmon [46] are of interest because they capture the transition for a fixed interelectrode gap distance and variable deposited energy. In particular, in the study of Lefkowitz et al. the discharge was used for ignition of methane-air mixtures and the authors noted little difference in terms of ignition between the high energy conditions (points corresponding to $\Pi_{t r}$ of 85 and 144 in Figure 13). However, for the low energy kernel (corresponding to $\Pi_{t r}=36$ ), the flame developed significantly slower. This suggests that ignition does not depend solely on the deposited energy and that the hydrodynamic regime can have an important impact on ignition. The results from Pannier [47] are also interesting because they were obtained with an NRP discharge operated in $\mathrm{CO}_{2}$. Therefore, the dimensionless number derived here can be applied to other gas mixtures 
as long as the fraction of energy deposited by ultra-fast heating is determined. The study of Kono et al. [34] focuses solely on toroidal kernel and that of $\mathrm{Xu}$ et al. [33] on diffusive kernels. Both results are well predicted by the dimensionless number.

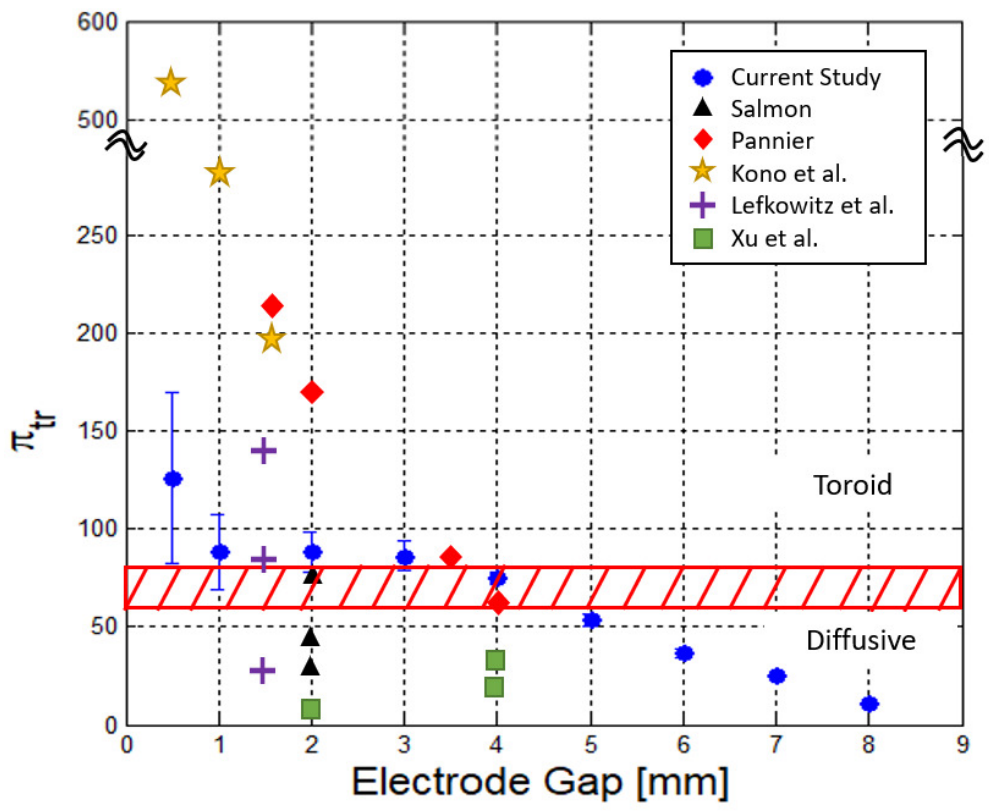

Figure 13: Dimensionless number predicting the transition between a toroidal and a diffusive kernel. The hatched region represents the uncertainty in the transition based on comparison between CFD and various experimental results.

It is important to note that there are two hidden variables inside the proposed dimensionless parameter: 1) a timescale corresponding to ultra-fast heating and 2) the nature of the mixture in which the discharge takes place. Both parameters are implicitly incorporated in the numerator, $E_{u h}$. This energy corresponds only to the energy converted into heat by the ultrafast heating mechanism, i.e. the energy coupled into the kernel before the shock wave detachment takes place ( $\sim$ hundreds of nanoseconds after the pulse). Only this energy plays a role in the hydrodynamics observed in the postdischarge because it is this energy that dictates the strength of the blast. Subsequently, the fraction of energy converted into ultrafast heating depends on the chemical kinetics of the gas mixture. This implies that the numerator must be tuned accordingly for various discharge pulse lengths and gas mixtures.

The pressure of the surrounding, $p_{0}$, is also an important parameter because the pressure ratio between the blast wave and the surrounding gas determines the strength of the shock which, in turn, influences the amount of vorticity generated in the post-discharge. This is illustrated experimentally in Figure 14. By lowering the pressure inside the reactor from 1 bar (Fig. 14-top) to 100 mbar (Fig. 14bottom), it is possible to bring the kernel from a diffusive regime into a toroidal regime. 

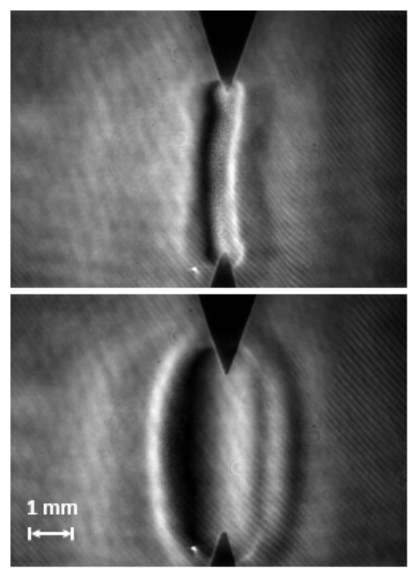

$(2 \mu s)$
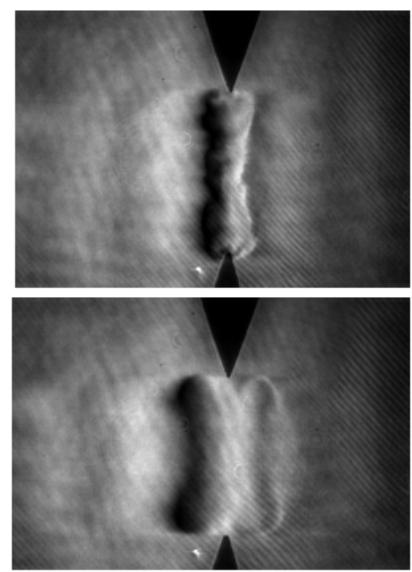

$(12 \mu s)$
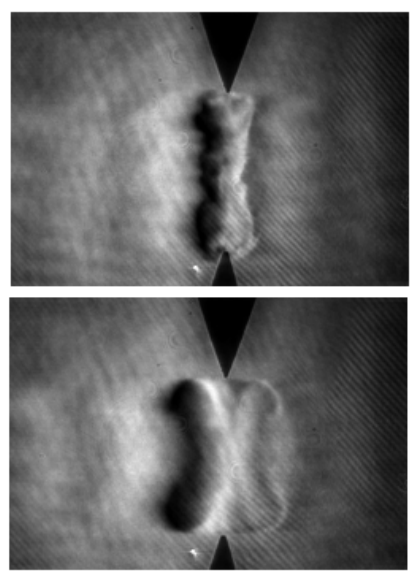

$(22 \mu s)$


$(32 \mu s)$

Figure 14: Experimental schlieren images of an NRP discharge in air inside of a d=4 mm gap at $\mathrm{p}_{0}=1 \mathrm{bar}, \boldsymbol{\pi}_{t r}=64$ (top) and $\mathrm{p}_{0}=100 \mathrm{mbar}, \boldsymbol{\pi}_{\boldsymbol{t r}}=106$ (bottom). Each image frame has a field of view: $6 \mathrm{~mm}$ x $9 \mathrm{~mm}$.

Finally, the limitation of the current definition of the dimensionless number is that as $d \sim r$ the blast wave becomes spherical and no baroclinic torque is formed. Thus, the proposed dimensionless number should be used for kernel prediction only as long as $d$ is significantly larger than $r(e . g ., d>2-3 r)$.

\section{Conclusions}

We have presented here experimental and numerical results on the hydrodynamic behavior of the plasma kernel formed by NRP discharges in atmospheric air. Two main flow regimes were identified using high-speed schlieren imaging: a) for small inter-electrode gap distances $\left(d_{\text {gap }}<4 \mathrm{~mm}\right)$, the hot gas kernel produced by the discharge collapses to form a symmetric torus; b) for large gap distances ( $d_{\text {gap }} \geq 4 \mathrm{~mm}$ ), the kernel is diffusive. Numerical modeling was successfully used to replicate the experimentally observed phenomena. With the aid of CFD, we were able to understand the mechanism responsible for vorticity generation, namely the baroclinic torque generated due to the misalignment of the density and pressure gradients in the flow at the moment of blast wave detachment. It is interesting to study further the impact of the two different hydrodynamic regimes on combustion ignition. A purely diffusive kernel would maintain a higher temperature for longer time and the highly reactive species generated during the discharge will have a longer residence time inside the channel. On the other hand, the smaller gap facilitates the formation of higher temperature/more reactive ignition kernels, which can propagate over larger distances with high speeds (e.g. $30 \mathrm{~m} / \mathrm{s}$ ) [48]. For flame stabilization, the mechanism of vorticity generation can be used to tailor the plasma kernel profile in order to augment stabilization. 


\section{ACKNOWLEDGMENTS}

This research was funded by the French National Research Agency (ANR) under project ASPEN (ANR-16-CE30-0004) and project PASTEC (ANR-16-CE22-0005).

\section{REFERENCES}

[1] Roupassov D V., Nikipelov A A, Nudnova M M and Starikovskii A Y 2009 Flow Separation Control by Plasma Actuator with Nanosecond Pulsed-Periodic Discharge AIAA J. 47 168-85

[2] Pilla G, Galley D, Lacoste D a, Lacas F, Veynante D and Laux C O 2006 Stabilization of a Turbulent Premixed Flame Using a Nanosecond Repetitively Pulsed Plasma Plasma Sci. IEEE Trans. 34 2471-7

[3] Choe J and Sun W 2018 Blowoff hysteresis, flame morphology and the effect of plasma in a swirling flow J. Phys. D. Appl. Phys. 51365201

[4] Starikovskii A Y 2005 Plasma supported combustion Proc. Combust. Inst. 30 II 2405-17

[5] Pancheshnyi S V., Lacoste D A, Bourdon A and Laux C O 2006 Ignition of propane-air mixtures by a repetitively pulsed nanosecond discharge IEEE Trans. Plasma Sci. 34 2478-87

[6] Lefkowitz J K and Ombrello T 2018 Reduction of flame development time in nanosecond pulsed high frequency discharge ignition of flowing mixtures Combust. Flame 193 471-80

[7] Kim W, Mungal M G and Cappelli M A 2008 Formation and role of cool flames in plasmaassisted premixed combustion Appl. Phys. Lett. 92 2006-9

[8] Barbosa S, Pilla G, Lacoste D a, Scouflaire P, Ducruix S, Laux C O and Veynante D 2015 Influence of nanosecond repetitively pulsed discharges on the stability of a swirled propane/air burner representative of an aeronautical combustor Philos. Trans. R. Soc. A Math. Phys. Eng. Sci. 37320140335

[9] Mintusov E, Serdyuchenko A, Choi I, Lempert W R and Adamovich I V. 2009 Mechanism of plasma assisted oxidation and ignition of ethylene-air flows by a repetitively pulsed nanosecond discharge Proc. Combust. Inst. 32 II 3181-8

[10] Bao A, Utkin Y G, Keshav S, Lou G and Adamovich I V. 2007 Ignition of ethylene-air and methane-air flows by low-temperature repetitively pulsed nanosecond discharge plasma IEEE Trans. Plasma Sci. 35 1628-38

[11] Lovascio S, Ombrello T, Hayashi J, Stepanyan S, Xu D, Stancu G D and Laux C O 2017 Effects of pulsation frequency and energy deposition on ignition using nanosecond repetitively pulsed discharges Proc. Combust. Inst. 36 4079-86

[12] Lovascio S, Hayashi J, Stepanyan S, Stancu G D and Laux C O 2018 Cumulative effect of successive nanosecond repetitively pulsed discharges on the ignition of lean mixtures Proc. Combust. Inst. 000 1-8

[13] Castela M, Stepanyan S, Fiorina B, Coussement A, Gicquel O, Darabiha N and Laux C O 2017 A 3-D DNS and experimental study of the effect of the recirculating flow pattern inside a reactive kernel produced by nanosecond plasma discharges in a methane-air mixture Proc. Combust. Inst. 36 4095-103

[14] Thiele M, Selle S, Riedel U, Warnatz J and Maas U 2000 Numerical simulation of spark ignition including ionization Proc. Combust. Inst. 28 1177-85

[15] Maly R and Vogel M 1979 Initiation and propagation of flame fronts in lean CH4-air mixtures 
by the three modes of the ignition spark Symp. Combust. 17 821-31

[16] Dumitrache C, Wilvert N, Yalin A and Shneider M 2013 Laser plasma formation using dual pulse pre-ionization 44th AIAA Plasmadynamics and Lasers Conference

[17] Settles G S 2001 Schlieren and Shadowgraph Techniques: Visualizing Phenomena in Transparent Media (Berlin: Springer-Verlag)

[18] Dumitrache C 2017 NOVEL LASER IGNITION TECHNIQUE USING DUAL-PULSE PREIONIZATION (Colorado State University)

[19] Chase M W 1998 NIST-JANAF Themochemical Tables, Fourth Edition J. Phys. Chem. Ref. Data, Monogr. 9 1-1951

[20] Srivastava B N and Srivastava R C 1959 Thermal conductivity and eucken correction for diatomic gases and binary gas mixtures J. Chem. Phys. 30 1200-5

[21] Roe P L and Pike J 1984 Efficient construction and utilisation of approximate Riemann solutions Comput. methods Appl. Sci. Eng. VI 499-518

[22] Toro E 1999 Riemann solvers and numerical methods for fluid dynamics (Springer-Verlag)

[23] Edwards J R 1997 A low-diffusion flux-splitting scheme for Navier-Stokes calculations Comput. Fluids 26 635-59

[24] Pai D Z, Lacoste D a and Laux C O 2010 Nanosecond repetitively pulsed discharges in air at atmospheric pressure-the spark regime Plasma Sources Sci. Technol. 19065015

[25] Pai D Z 2008 Nanosecond Repetitive Pulsed Plasma in Preheated Air at Atmospheric Pressure (PhD Thesis, Ecole Centrale Paris)

[26] Popov N A 2001 Investigation of the mechanism for rapid heating of nitrogen and air in gas discharges Plasma Phys. Reports 27 886-96

[27] Rusterholtz D L, Lacoste D A, Stancu G D, Pai D Z and Laux C O 2013 Ultrafast heating and oxygen dissociation in atmospheric pressure air by nanosecond repetitively pulsed discharges $J$. Phys. D. Appl. Phys. 46464010

[28] Stancu G D, Janda M, Kaddouri F, Lacoste D A and Laux C O 2010 Time-resolved CRDS measurements of the N2(A) density produced by nanosecond discharges in atmospheric pressure nitrogen and air J. Phys. Chem. A 114 201-8

[29] Stancu G, Kaddouri F, Lacoste D and Laux C 2009 Investigations of the Rapid Plasma Chemistry Generated by Nanosecond Discharges in Air at Atmospheric Pressure Using Advanced Optical Diagnostics AIAA 2009-3593 pp 1-7

[30] Lo A, Cessou A, Lacour C, Lecordier B, Boubert P, Xu D A, Laux C O and Vervisch P 2017 Streamer-to-spark transition initiated by a nanosecond overvoltage pulsed discharge in air Plasma Sci. Technol. 26 593-600

[31] Minesi N, Stepanyan S, Mariotto P B, Stancu G-D and Laux C O 2019 On the arc transition mechanism in nanosecond air discharges AIAA 2019-0463 (San Diego: AIAA Scitech) p 9

[32] Xu D 2013 Thermal and hydrodynamic effects of nanosecond discharges in air and application to plasma-assisted combustion (PhD Thesis, Ecole Centrale Paris)

[33] Xu D A, Shneider M N, Lacoste D A and Laux C O 2014 Thermal and hydrodynamic effects of nanosecond discharges in atmospheric pressure air J. Phys. D. Appl. Phys. 47

[34] Kono M, Niu K, Tsukamoto T and Ujiie Y 1988 Mechanism of flame kernel formation produced by short duration sparks Symp. Combust. 22 1643-9

[35] Thompson K W 1987 Time dependent boundary conditions for hyperbolic systems J. Comput. Phys. 68 1-24

[36] Poinsot T J and Lelef S K 1992 Boundary conditions for direct simulations of compressible viscous flows J. Comput. Phys. 101 104-29

[37] Leonov S B, Isaenkov Y I, Firsov A A, Nothnagel S L, Gimelshein S F and Shneider M N 2010 
Jet regime of the afterspark channel decay Phys. Plasmas 17

[38] Kundu P K and Cohen I M 2008 Fluid Mechanics (New York: Academic Press)

[39] Dumitrache C and Yalin A P 2018 Numerical Modeling of the Hydrodynamics Induced by DualPulse Plasma 2018 AIAA Aerospace Sciences Meeting pp 1-12

[40] Bradley D, Sheppard C G W, Suardjaja I M and Woolley R 2004 Fundamentals of high-energy spark ignition with lasers Combust. Flame 138 55-77

[41] Dumitrache C, Vanosdol R, Limbach C M and Yalin A P 2017 Control of Early Flame Kernel Growth by Multi-Wavelength Laser Pulses for Enhanced Ignition Sci. Rep. 7

[42] Morsy M H 2012 Review and recent developments of laser ignition for internal combustion engines applications Renew. Sustain. Energy Rev. 16 4849-75

[43] Singh B, Rajendran L K, Gupta P, Scalo C, Vlachos P P and Bane S P 2019 Experimental and Numerical Study of Flow Induced by Nanosecond Repetitively Pulsed Discharges 1-15

[44] Moumouni A S 2017 Experimental Study of Hydrodynamic Effects of Nanosecond Repetitive Pulsed (NRP) Discharges in Plasma-Flame Interaction (ENSMA Poitiers)

[45] Lefkowitz J K, Guo P, Ombrello T, Won S H, Stevens C A, Hoke J L, Schauer F and Ju Y 2015 Schlieren imaging and pulsed detonation engine testing of ignition by a nanosecond repetitively pulsed discharge Combust. Flame 162 2496-507

[46] Salmon A 2018 Diagnostics and kinetics of reactive oxygen and nitrogen species in atmospheric pressure non-equilibrium discharges for bio-decontamination (CentraleSupelec)

[47] Pannier E 2019 Conversion of Carbon Dioxide with Nanosecond Pulsed Discharges (CentraleSupelec)

[48] Stepanyan S, Hayashi J, Salmon A, Stancu G D and Laux C O 2017 Large-volume excitation of air, argon, nitrogen and combustible mixtures by thermal jets produced by nanosecond spark discharges Plasma Sources Sci. Technol. 26 